\title{
Ueber einige karntnerisch-salzburgische Privaturkunden
} des 11. und 12. Jahrhunderts.

\author{
Von
}

\section{Oswald Bedlich.}

Die Entwicklung des deutschen Privaturkundenwesens bis in das 13. Jahrhundert ist auf mannigfach schwankenden Wegen vor sich gegangen. Dem Bedürfniss nach urkundlicher Aufzeichnung und Sicherung, das sich schon von der Mitte des 11. Jahrhunderts an immer deutlicher fühlbar machte, und dem keine gefestete Einrichtnng wie das Notariatswesen Italiens entgegenkam, strebte man in verschiedener Weise abzuhelfen. Die Hauptmomente und Factoren dieses Entwicklungsganges versuchte ich für Baiern in der Abhandlung über bairische Traditionsbücher und Traditionen zn charakterisiren '), allein der Stoff war zu gross, um da ins einzelne gehen zu können. Die folgenden Erörterungen sollen nun einen kleinen Beitrag zar Vervollständigung dieses Bildes bieten. Sie betreffen einige Urkunden im Besitze des Kärntner Geschichtsvereins, auf die ich gelegentlich der Studien über Traditionsbücher aufmerksam geworden war. Dank der zuvorkommenden Güte der Direction des genannten Vereins ward ich in den Stand gesetzt, diese Stücke eingehend untersuchen zu können und es zeigte sich nun an denselben eine ganze Reihe von interessanten Erscheinungen, von denen ich einige bereits in jener Abhandlung erwähnt habes), während bei einer Anzahl von andern, die durch ibre Gleichartigkeit zusammenhängen, eine gesonderte und etwas ausführlichere Besprechung nicht unangemessen erschien.

Als ältestes Stück in dieser Reibe erscheint eine Urkunde, die eine solche Fülle unverträglicher Widersprüche aufweist, dass man auf den ersten Blick schon sagen kann, so wie sie vorliegt und entstan-

1) Mitth. des Instituts 5, 1 ff., besonders $64 \mathrm{ff} . \quad$ 2) A. ar $0.72 \mathrm{f}$.

Mittheilungen $v$. 
den zu sein behauptet, könne sie unmöglich entstanden sein. Es ist die Beurkundung der Weihe und Bewidmung der von einem Freien Tessina, genannt Rapoto, zu Glantschach in Kärnten erbauten Andreaskirche durch Erzbischof Friedrich von Salzburg 1). Auf steifem Pergament von einer Hand geschrieben beginnt sie: Anno dominicę incarnationis deccc ${ }^{\mathrm{mo}}$ quidam nobilis vir Tessina cognomine Rapoto . . quandam domum ad laudem dei in loco Globzach consensu et licentia Friderici archiepiscopi edificavit. Unter dem Texte steht in vollständiger Nachahmung von Königsurkunden in verlängerter Schrift mit ausgeführtem Monogramm: Signum domni Arnolfi (M) piissimi regis, ron gleicher Hand und Tinte wie der Context geschrieben. Am untern Rand der Urkunde mehr gegen rechts ist das Siegel Erzbischof Friedrichs in der Weise aufgedrückt, dass der untere Wulst über den Rand des Pergaments hinausragt, auf dessen Rückseite übergreift, uud mit dem rückwärtigen, jedenfalls durch eiuen Schnitt durchgedrückten Wachsklumpen eine Masse bildet.

Die Urkunde soll uns also glauben machen, dass im Jahre 900 Erzbischof Friedrich von Salzburg diese Kirche geweiht und eine über Weihe und Ausstattung gefertigte Urkunde mit seinem Siegel bekräftigt habe und dass dieselbe von König Arnolf mit seinem Namenszeichen unanfechtbar gemacht worden sei.

Das heisst uns viel zugemuthet. Erzbischof Friedrich hatte von 958-991 das Erzbisthum Salzburg inne, Kaiser Arnolf war bereits 899 gestorben. Dass als; das Signum Arnolfs und das Jahr 900 mit der Handlung gar nichts zu thun haben, bedarf wol keiner weiteren Worte. Diese Behauptung aber, dass Arnolf und Erzbischof Friedrich im Jahre 900 beide gelebt haben sollen, zeigt auch schon, dass das Stück in dieser Gestalt auch nicht unter Erzbischof Friedrich entstauden sein kann, denn es ist doch geradezu undenkbar, dass man die Beurkundung seiner eigenen Handlung ins Jahr 900 bätte setzen und er selbst dies mit seinem Siegel hätte bekräftigen wollen und und können. Dass dies aus Cnwissenheit, oder, trotz eigenem besseren Wissen mit Absicht auf die Unwissenheit der mdern rechnend, geschehen konnte, weist bereits entschieden auf eine viel spätere Zeit der Entstehung hin. Und damit stimmt denn auch die Schrift. Sie gehört ausgesprocheu erst der zweiten Hälfte des 11. Jahrhunderts an; sie trägt im ganzen den Charakter der Urkunden-Minuskel, wie sie nicht bloss in Diplomen, sondern auch in den Urkunden der

1) Vgl. Ankershofen. Gesch. Kärntens 2, 530. Daselbst Reg. u. Urk. 37 n. 44 ist das Stũck gedruckt, doch nach einem Gurker Copialbuch aus dem 18. Jahrbundert, in welchem das gleich zu erwähnende Signum Arnolfs fehlt. 
weltlichen und geistlicben Fürsten dieser Zeit angewendet wird, ja auch vielfuch in Traditionscodices, so in denen von Brixen, Ebersberg, S. Emmeram, Formbach u. a. erscheint ${ }^{1}$ ).

Und nun das Siegel Erzbischof Friedrichs. Gegen seine Echtheit lässt sich durchaus nichts einwenden, wie es denn auch Richter, Die ältesten Siegel der Salzburger Erzbischöfe, Mitth. d. Centralcommission VIII, CXXI (1882) als unzweifelhaft echt behandelt ${ }^{2}$ ). Das Siegel musste somit einer Urkunde Erzbischof Friedrichs entnommen worden sein ${ }^{3}$ ). Für eine solche Ablösung eines schon anf einer andern Urkunde aufgedrückten Siegels und seine neuerliche Verwendung dürfte auch die bereits erwähnte, ganz eigenthümliche Befestigung sprechen, die ich, so vielfach sonst die Arten derselben bei Privaturkunden wechseln, doch nirgends beobachtet habe: da bei der Ablösung aus der alten Urkunde der rückwärtige Fuss des Siegels abgebrochen werden musste, suchte man bei der zweiten Verwendung die Haltbarkeit dadurch zu verstärkeu, dass man nicht bloss einen neuen Fuss rückwärts daraufgoss, sondern auch noch diese rückwärtige Wachsmasse mit der vordern verschmolz.

Die naheliegende Frage, von welcher Urkunde Erzbischof Friedrichs das Siegel genommen wurde, führt uns zugleich auf die Frage nach der Vorlage für die uns erhaltene Form. Eine solche werden wir sicher anzunehmen haben, eine wirklich unter Friedrich ausgestellte Urkunde üher den Bau, die Weihe und Ausstattung der Andreaskirche zu Glantschach. Denn gegen diese zu Grunde liegenden Thatsachen selbst lässt sich jat gar nichts einwenden und Ankershofen, der unser Document nur aus einem Copialbuch kannte und deshalb das Jahr 900 leiclit als einen Schreibfehler ansehen durfte, nimmt sie auch ohne Bedeuken in seine Geschichte Kärntens auf. Dieser Vorlage werden ohne Zweifel die sachlichen Angaben und die Zeugen angehören und ihr wird, wie es gewiss wahrscheinlich ist, auch das Siegel des Erzbischofs abgenommen worden sein.

1) Vgl. Urk. des Markgralen Ernst c. 1075 M. graphica j tab. 3, Herzog Heinrichs von Kärnten 1103 ib. tab. 5, Bischof Reginmars von Passau 1125 ib. 7 tab. 1 ; vgl. auch Mitth. d. Instituts $5, i 7$.

2) Die Abbildung (Fig. 1) zeigt kleine Ungenauigkeiten. Ueber GRA fehlt der Abkürzungsstrich, der Bischofstab erreicht mit seiner Rundung zwischen GRA und EPS den Rand des Siegelfeldes, während dies im Siegel selbst nntex $\mathbf{P}$ der Fall ist.

3) Eine Nachahmung (Nachguss) des Siegels, wie eine solche Buchwald, Bischofs- und Fürstenurkunden 17 für das Mittelalter in sehr ausgedehntem Masse annimmt, setzt natürlich auch immer ein Muster voraus und würde in unserem Falle keine bessere Ėrblärung bieten können. 
Allein die Form der uns vorliegenden Fassung ist dieser eigenthümlich und sie gewährt uns nun Anhaltspuncte, um auch die Entstehnngszeit mit hoher Wahrscheinlichkeit bestimmen zu können. Der Eingang mit dem Jahre 900 ist selbstrerständlich, wie schou erwähnt, erst neu hinzugefügt und weist auf eine Erzbischof Friedrich weit fernliegende Zeit. Näher der Sache bringen uns aber folgende Stellen: (Tessina) .. rectam decimationem in manus archiepiscopi legitime tradidit et eiusdem decimę terciam partem cum duabus hǒbis . . concambivit et eidem ęcclesię ius baptizandi et sepeliendi . . obtinuit, ordinante et confirmante hoc archiepiscopo, ne aliquis succedentium episcoporum commutare potestatem habeat. Diese deutliche Betonung der recta decimatio wäre unter Friedrich und seinen nächsten Nachfolgern etwas ganz ungewöhnliches; sie wird erst verständlich und möglich mit der Zeit, da die Salzburger Erzbischöfe ihre Bemühungen begannen, die decima consuetudinaria, d. i. den ein für alle mal bestimmten, nicht nach dem Ertrag der Ernte bemesseneu Zehent, wie er zuerst nur den Slaven gewährt worden, daun aber allmälig landesüblich und auch von den deutschen Diöcesanen stillschweigend angenommen worden war, zu verdrängen und den eigentlichen, richtigen und canonischen Zehent, die iusta, recta, integra oder auch acquisitoria decima von den Bewohnern des Erzhisthums zu erlangen. Vor allem Gebhard (1060-1088) war in dieser Hinsicht mit Erfolg thätig, auf einer eigenen Synode zu Maria Saal sind um das Jahr 1065 diese Zehentverhandlungen durchgeführt worden ${ }^{1}$ ). Seit dieser Zeit wird denn auch die neue Art des Zehents betont; ja es erscheint sogar ein bestimmtes Formular für die Zehentübergabe und Zehentablösung, sowie für die Bestätigung durch den Erzbischof. Eine Urkunde ron c. 1075 , Steierm. CB. 1, 96 n. 79, sagt uns, dass der Freie Bartnit ex omnibus prediis suis que in episcopio Juvavensi habuit, rectam decimationem in manum archiepiscopi Gebahardi legitime tradidit, dass er die Kirche $S$. Loreuzen bei Knittelfeld abtrat und cum eadem ęcclesia . . redimebat et concambiebat decimationem .. ad ęcclesiam suam Fiustriza constractam et in eadem ęcclesis ius baptizandi sępeliendique .. obtinuit, ordinante hoc et confirmante archiepiscopo Gebahardo ea ratione, ut nullus succedentium episcoporum hoc pactum mutare aut infringere potestatem habeat. In den gleichen: nur unbedeutend variirenden Wendungen bewegt sich die Urkunde Marquards von Eppenstein c. 1066, Steierm. UB. 1, 77 n. 68, die ebenfalls Zehentangelegenheiten betrifft.

1) Vgl. Ankershofen, Gesch. Kärntens 2, : 54, 945. Mayer, Die östl. Alpen. länder im Investiturgtreite $82 \mathrm{f}$. 
Die schlagende Uebereinstimmung der Formeln dieser unter Gebhard entstandenen Urkunden mit unserem fraglichen Stincke ist wol ohne weiteres klar. Wir werden demnach, da ja anch der Schriftcharakter damit harmonirt, seine Entstehung ohne grosses Bedenken in diese Zeit, unter Erzbischof Gebhard setzen und werden in dem Wörtchen recta decimatio den eigentlichen Grund za dieser neuen Ausfertigung suchen dürfen, welche die Sache getreulich wiedergibt, aber mit dem gerade für solche Urkunden aufgekommenen Formulare umkleidet und, was eben in der Vorlage sus Erzbischof Friedrichs Zeit gewiss nicht gestanden ist, dem nunmehr geforderten canonischen Zehent die gewünschte Betonung verschafft.

Es bleibt uns schliesslich noch die Signumzeile Arnolfs zu erörtern. Wie schon bemerkt, ist sie von gleicher Hand und Tinte wie der Text der Urkunde, wir haben also eine Nachzeichnung vor uns. Es ist ihr die verlängerte Schrift eines Diploms von Arnolf, besonders bei einzelnen Buchstaben wie $o, r, f$, auch $g$ und $s$, so ziemlich gelungen. Das Monogramm ist ungeschlacht, aber richtig. Noch merkbare Spuren ron früher vorhandenen, dann radirten Buchstaben, die eine höhere Lage des Monogramms ergeben würden, zeigen, dass ein erster Versuch missglückt war. Dem entspricht auch die dem jetzigen Monogramm gegenüber viel zu hohe Stellung der vorhergehenden Worte signum domni Arnolfi, während das nachfolgende piissimi regis demselben mehr angepasst ist. Das aber lässt diese Signumzeile unbedingt schliessen, dass ibr Schreiber ein Originaldiplom Arnolfs vor sich gehabt haben muss. Welches jedoch dies gewesen ist, scheint nicht mehr festgestellt werden zu können. Denn die einzige Signumzeile in einem Diplom Arnolfs von salzburgisch-kärntnerischer Provenienz, welche die Formulirung S. d. A. piissimi regis besitzt, nämlich in der Urkunde für den Cleriker Sigibold 888 März 19, Juvavia Anh. 106, Orig. im Staatsarchiv in Wien, ist ganz entschieden von einer andern Hand, als die ron unserm Nachzeichner so ängstlich copirte. Doch es genügt ja, überhaupt eine solche Nachbildung einer königlichen Signumzeile in einer Privaturkunde des 11. Jahrhunderts constatirt zu haben und wenn wir um die Absicht fragen, welche den Schreiber hiebei geleitet haben mochte, so werden wir sie einfach in dem Wunsche zu suchen haben, der Urkunde womöglich die beste Beglaubigung zu verschaffen. Wie Ficker Beiträge 1, 281 Fälle bringt, wo wirklich an Privaturkunden ein von der königlichen Kanzlei ausgegangenes Schlussprotokoll ohne jede vorhergehende Ankündigang zugefügt wurde, so ist auch hier der Gedanke massgebend gewesen, wenigstens durch den Schein der Unterzeichnung irgend eines Königs, 
von dem ein Diplom als Muster gerade bequem vorlag, der Urkunde unanfechtbare Geltung zu sichern - freilich mit Hilfe einer groben Unwahrheit, als welche dieser Theil und das damit sichtlich im engsten Zusammenhange steheude Jahr 900 allerdings bezeichnet werden muss.

Der ganze Fall würde sich also zusammenfassend dahin charakterisiren lassen. Eine unter Erzbischof Friedrich (958-991) über die von ihm vollzogene Weihe und über die erste Bewidmung der Andreaskirche zu Glantsehach ausgestellte und von ihm besiegelte Urkunde ward hundert Jahre später unter Erzbischof Gebhard sehr wahrscheinlich ans Anlass der allgemeinen Zehentregulirung in der Salzburger Erzdiöcese, vielleicht auf directen Befehl des Erzbischof's und wol sicher in dessen Umgebung neu ausgefertigt; man umgab den sachlichen Kern der alten Urkunde mit den für solche Fälle gerade üblich gewordenen Formeln, vermied aber sorgfältig jede absichtliche Andeutung einer so viel späteren Entstehung und nahm demgemäss auch das Siegel Erzbischof Friedrichs mit herüber. Ja, man suchte durch die Einfügung des Jahres 900 und der nachgeahmten Signumzeile König Arnolfs auch noch das Zeitalter Friedrichs viel weiter zurückzuversetzen, und der Urkunde ein bedeutend ehrwürdigeres Alter und eine um so stärkere Glaubwürdigkeit zu verschaffen. So ist ein Document entstanden, voll äusserer und innerer Widersprüche, dennoch aber seinem Inhalt nach echt und in der bezeugten Thatsache glaubwürdig. Ein, unechtes Original ", oder mit Buchwald zu reden ein "Authenticat", oder eigentlich, da absichtliche Verunechtungen darin nachzuweisen sind, ein „interpolirtes Authenticat“, wenn man schon ein Wort dafür haben will.

Ebenfalls mit diesen Zehentangelegenheiten im Zusammenhauge steht ein zweiter Fall. Die Aebtissin R(ichardis) von Göss übergibt dem Erzbischof Geblıard die Hälfte ihrer Kirche zu Sörg und andern Besitz und erhält dafür die Zehenten zurück, welche sie ihm früher abgetreten hatte, sowie die Pfarrechte für die Kirchen zu Göss und Sörg. Die Urkunde ist nach dem Authenticum des Gurker Domcapitelarchivs (jetzt als Depositum in Klarrenfurt) im Steierm. UB. 1, 80 n. 69 zu c. 1070 gedruckt und Zahn bemerkt dazu S. 81 Anm. 2: "Diess ohne Zweifel die Urkunde, welche in der Entscheidung Erzbischofs Konrad III. von Salzburg von 1178 1. März, Frisach, als falsch behandelt wurde, was aus ihrer Schrift sich nicht behaupten lässt. Es hatte sich nämlich, wie in dieser Eutscheidung (Steierm. UB. 1, 558 n. 593) berichtet wird, die Aebtissin Adelheid von Göss an Erabischof Konrad mit der Klage geweudet, dass die Dowherreu von Gurk sich in gauz ungerechter Weise das Patronat der S. Martins- 
kirche zu Sörg aumassen, und Aebtissin und Convent hatten auf der Provinzialsynode, die der Erzbischof im Herbst 1177 zu Frisach hielt, ein Privilegium producirt, cuius ratione petitionem capellę ad se pertinere asserebant. Der Erzbischof beauftragte drei Geistliche mit der Prüfung dieser Orkunde, qui diligenti examinatione illud perscrutantes et falsitatem eius deprehendentes palam nobis prodiderunt. Zudem brachte das Domcapitel eine Reihe von Zeugen dafür bei, dass Sörg jedenfalls schon seit mehr als 40 Jahren zur Pfarre Glautschach gehört habe und dem Domcapitel von Gurk das Patronat zugestanden sei. Die Nonnen vou Göss waren überführt, Sörg blieb von da an eine Filiale der Pfarre Glantschach und das Gurker Domcapitel behielt unbestritten das Patronat dieser Kirche. Es ist sehr schade, dass die Gründe für das Erkenntniss auf Fälschung nicht angegeben sind. Wir können sie aber allerdings aus dem Bestande dieser fraglichen Urkunde - denn ohne Zweifel ist sie mit unserem Stücke identisch noch genügend entwickeln.

Zunächst muss als ein solcher Grund die Schrift betrachtet werden, im Gegensatz zu Zahus angeführter Bemerkung. Sie trägt so entschieden deu ausgeprägten Charukter des 12 . Jahrhunderts an sich, dass ein unbefangener Beurtheiler, der nicht durch den Inhalt beeinflusst ist, sie ohne weiteres zum mindesten der Mitte dieses Säculums zuweisen wird. Ein ferneres auffälliges Moment ist die verrätherische Wendung abbatissa de Gosse $R$. ex omnibus prediis ad monasterium, cui preerat, pertinentibus . . tradidit. Zu Lebzeiten der Aebtissin Richardis kann dies cui preerat gewiss nicht geschrieben sein. Der Hauptgrund aber zur Verdächtigung dieser Urkunde, der auch sicherlich bei jenem folgenreichen Erkenntniss den Ausschlag gegeben hat, liegt im Siegel. Denn nicht das Siegel Erzbischof Gebhards, das nach der Corroborationsformel ordinante hoc et legitime confirmante archiepiscopo Gebehardo zu erwarten stände, oder das der Aebtissin Richardis, das auch noch möglich wäre, ist es, sondern das Siegel der Aebtissin Adelbeid, eben derjenigen, welche die Ansprïche auf die S. Martinskirche wieder geltend machen wollte. Zahn sagt bloss „aussen unten eingehängtes Siegel der Aebtissin " und es wird uatürlich jedermann, der die Crkunde nicht selbst sieht, dies auf Richardis deuten. Allein auf dem allerdings sehr abgeschliffenen und verletuten Siegel ist doch noch von der Legende deutlich zu lesen: .. LHEDIS DI GRA GOSSENSIS . . ABBATISSA ${ }^{1}$ ). Und somit hätten

1) lch bemerke noch die eigenthümliche Art der Besiegelung. Für das Siegel wurde rechts unten am Pergament ein eigener Fortatz gelassen, der beim $\mathrm{Zu}$ - 
die drei geistlichen Diplomatiker von 1177 Recht gehabt: Die Urkunde, die ein Siegel Erzbischof Gebhards oder etwa der Aebtissin Richardis hätte tragen sollen, trug eines der hundert Jahre später lebenden Aebtissin Adelheid, also war sie falsch.

Und dennoch behaupte ich, den Nonnen von Göss ist damit einigermassen Unrecht geschehen. Freilich, gegen die auf der Synode zu Frisach 1177 angeführten Zeugschaften lässt sich nichts einwenden und es scheint allerdings das Kloster Göss die Ausübung seiner Rechte an der Pfarre Sörg durch geraume Zeit völlig vernachlässigt zu haben. Aber die von ihm producirte, für falsch erklärte Urkunde ist doch nur wieder eine, freilich unberufene Neuberstellung einer wirklich unter Erzbischof Gebhard entstandenen Urkunde. Vor allem ist es die Fassung, welche mir dies zu beweisen scheint. Sie stimmt von Anfang bis zu Ende genau mit dem Formular überein, das wir bei unserem ersten Falle kennen lernten, das unter Gebhard für solche Zehenturkunden und Pfarrrechtverleihungen angewendet wurde. Sie stimmt geradezu wörtlich mit der Urkunde Hartnits, die wir bereits oben citirten. Sodann fehlen auch nicht anderweitige Zeugnisse für die Zugehörigkeit der Martinskirche zu Göss. Wie schon Meiller, Regesten der Salzb. Erzbischöfe 489 Anm. 9 hervorhebt, ist in den Bestätigungsbullen der Päpste Eugen III. 1148 Apr. 13 und Gregor IX. 1230 Mai 2 die ecclesia s. Martini de Soriche ausdrücklich unter den Besitzungen des Klosters aufgeführt. Allerdings waltet nun bezüglich der ersten Bulle ebenfalls ein eigenthümliches Verhältniss ob. Sie ist nach Zahn, Steierm. UB. 1, 289 Anm. 1 ein „rescribirtes Original ", das heisst, eine im Kloster selbst hergestellte Abschrift, die dem Original in Inhalt und Form nachgebildet wurde mit der offenkundigen Absicht, sie eben als Original zu gebrauchen. Inhaltlich kann sie uns also auch das Original repräsentiren und weder gegen Inhalt noch gegen die Formeln kann irgend ein Einwand erhoben werden. Zahn macht dabei auf die für uns höchst interessante Thatsache aufmerksam, dass in einer Urkunde ron 1188 Juni 7, Steierm. UB. 1, 674 n. 690, eine Nonne von Göss Namens Perhta erwähnt wird, der man eine durch Feuer balb zerstörte Bestätigungsurkunde Erzbischof Adalberts, da ihr eiusdem privilegii tenor notissimus erat, reparandum tradidit. „Man scheint, bemerkt Ficker Beiträge 1, 32 gelegentlich eben dieses Falles, darin nichts anstössiges gefunden zu haben". Nach Perhtas Tode fand man jedoch dieses, "reparirte" Stück nicht vor.

aammenfalten heraufgeschlagcn wird. Durch je zwei übereinanderliegende kleine Schnitte gehen zwei achmale Pergamentstreifchen, an denen rückwärts die Siegel. masse angebracht wurde. 
Hätte die Nonne ihre Aufgabe durchgeführt, es wärde so gut, wie die zweite uns vorliegende Bestätigung des Erzbischofs gegolten haben; ihr war der Inhalt der beschädigten Urkunde gensu bekannt, sie genoss offenbar auch als Schreibkundige einen Ruf und galt als eine öffentlich anerkannte Vertrıuensperson, der man also sogar die selbstständige Wiederherstellung oder Neuherstellung von Urkunden anvertraute. Wir können eine solche Erscheinung geradezu als einen unbewussten und unvollkommenen Anlauf betrachten, in einer dem italienischen Notariatswesen ähnlichen Weise dem stark wachsenden Bedürfniss nach Schriftlichkeit des Rechtsverkehres zu entsprechen.

Es wird nicht zu gewagt sein, wenn wir dieser Perhta von Göss auch die Anfertigung unserer Urkunde zuschreiben. Wie bei der Urkunde Erzbischof Adalberts und der Bulle Eugens, die wir vielleicht auch auf ihre Rechnung setzen können ${ }^{1}$, wird auch hier die einfache $\mathrm{Ab}$ sicht gewesen sein, die alte Vorlage, die entweder eine einfache Traditionsnotiz ohne weitere Beglaubigung, oder viel wahrscheinlicher gleich der Urkunde Hartnits einst mit dem Siegel Erzbischof Gebhards versehen gewesen war, das verloren gegangen, in rechtskräftiger Gestalt wieder herzustellen. In unserem Falle können wir auch mit hoher Wahrscheinlichkeit den Streit mit Gurk und die Synode von Frisach als Veranlassung und Zeitpunct dieser neuen Ausfertigung bezeichnen. Die Aebtissin ist gewiss sine dolo vorgegangen, aber nicht glücklich. Hätte man noch das Siegel Gebhards besessen, die Orkunde wäre dann wol nicht beanstandet worden. Vielleicht hätte sie aber auch dann wider die erdrückenden Zeugenaussagen, denen man nichts entgegenstellen konnte, wenig geholfen.

An die Erörterung dieser Fälle möchte ich noch ein Stỉck anschliessen, das angeblich aus der Zeit Erzbischof Hartwigs von Salzburg (991-1023) herrühren soll und die Errichtung einer Kirche und Pfarre zu S. Martin im Krapfeld betrifft. Ankershofen kannte die Urkunde aus einer neueren Copie, brachte jedoch nur einen Auszug in seinen Urkunden u. Regesten zur Gesch. Kärntens, Arch. f. K. öst. GQ. 2, 349 n. 162 in Anschluss an die durch Erzbischof Gebhard 1075 Juli 16 beurkundete, von Bischof Gunther von Gurk ins Werk gesetzte Wiedererrichtung dieser Kirche und Erweiterung ihres Besitzes. Das fragliche Stück ist auf $31 \mathrm{~cm}$ breitem, $17.5 \mathrm{~cm}$ hohem Pergamentblatt geschrieben. Rechts oben hat der Schreiber einen viereckigen Raum absichtlich frei gelassen, als wenn ein Siegel hieher

1) Eine Vergleichung dieser Bulle im steirischen Landesarchiv mit unoerem Stücke könnte wol bestimmteren Aufschluss geben. 
bestimmt gewesen wäre. Das Siegel, das Erzbischof Hartwigs, befiudet sich aber unteu in der Mitte des leer gebliebeneu Raumes; es ist jetzt abgebildet bei Richter a. a. 0. CXXI Fig. 21). Die Urkunde beginnt wie die gewöhnlicben Traditionen: Notum sit omnibus Christi fidelibus .. qualiter quidam nobilis vir nomine Heimo . . ędificavit istam ecclesiam cum consensu et licencia venerabilis Juvavensis archiepiscopi Hartwici ac dedicata est . . anno idcec, indictione XIII, IIII. non. iulii; die Kirche erhält die pfarrlichen Rechte und Zehentbezug, was mit dem Gute Sulpa abgelöst wird, eo pacto et tenore, si quis ipsam aeclesiam huius aecclesiastici iuris per instinctum diaboli destitueret, anatbema esset et in perpetuum particeps existeret cum infidelissimo et nequissimo Juda. Darauf folgt noch die Aufzählung der gewidmeten Güter, Zehenten und Hörigen.

Ganz besonders auffällig ist nun wieder die Datirung. Was soll diese sonderbare Jahreszahl bedeuten? Wird man nicht an das Jahr 900 in der erstbesprochenen Urkunde gemahnt, an eine ganz willkürliche, nur recht weit zurückgreifendle Datirung. Wie hätte man so etwas schreiben können, wäre die Urkunde wirklich unter Erzbischof Hartwig entstanden. Ond nun kommt denn auch hier wieder die Schrift unsern Verdacht bestärkend hinzu. So schrieb man noch nicht um das Jahr 1000, die oben mit Ausatz beginnenden Schäfte von $b, h, l$, einzelne Buchstaben, so besonders $W$ weisen bereits auf die zweite Hälfte des 11. Jahrhunderts. Dazu tritt noch ein gesucht diplonatischer Charakter, der in verkünstelten aber ungeschlachten Schnörkeln und Verbindungen das Aussehen von Urkundenschrift zu erlangen strebt. Und endlich das Siegel. Glücklicherweise ist uns noch ein zweites Siegel Hartwigs erhalten, nämlich an der unbezweifelt echten Stiftungsurkunde des Klosters S. Georgen am Längsee, Authenticum im Archiv des Kärıtner Geschichtsvereins, gedruckt bei Ankershofen Gesch. Kärutens 2, Reg. u. Urk. 84 n. 39; diese

1) Auch hier sind kleine Berichtigungen nöthig. Nach ARCHIEPISCP fehlt das Abkürzungazeichen für VS; sodann ist die Abbildung viel zu schön geratheu, wäbrend das Siegel, wie wir gleich zeigen werden, eine plumpe und rohe Nachbildung ist. Es wăre also noch eine Reproduction des einzigen uns erhaltenen echten Siegels Hartwigs, das an der S. Georgener Urkunde sich befindet. nothwendig. - Es sei gestattet, hier gleich auch noch einiges über das Siegel Erzb. Balduins, p. CXXII Fig. 4 zu bemerken. Mir lag das an der Stiftungsurkunde des Nonnenklosters Gurk 1042, 104: befindlicbe vor: diesem gegenüber fehlt in der Abbildung der Abkürzungsstrich über EPS, der Bischolsstab ist nach aussen, anstatt nach innen gebogen, das Gewand hat am Halse (ganz wie hei Hartwig) einen viereckigen Ausschuitt, der der Abbildung tehlt, während ibr anscheinend runder Ausschnitt nur eine Falte ist. 
Urkuude dürfte auch noch weitere Winke zur Erklärung unseres fraglichen Stückes an die Hand geben. Das Siegel derselben ist uun, wie Richter CXXIII bemerkt, ,nicht ron derselben Stampiglie wie das erste, . . doch so ähnlich, dass nur genauere Untersuchung die Ungleichheit erkennen lässt". Allerdings, nur haben wir nicht zwei verschiedene Siegel Hartwigs vor uns, sondern ein echtes und eine Nachbildung. Denn die Ungleichheit besteht nicht in Verschiedenheiten der Form, sondern in Verschiedenheiten der Ausführung. Die Grösse beider Siegel ist die gleirhe, die Legende ist dieselbe, soweit sie beim Georgener Siegel noch erhalten ist. Allein das deutlich krause Haar bei diesem letztern, das in fünf Wülsten vortritt, ist beim andern geradezu zu fünf Knollen geworden; die Augen sincl hier unförmlich gross und glotzend, der Mund ist nicht ausgedrückt. Das über die Arme in gut gemachten Falten heribhängende Gewand im Georgener Siegel ist beim andern in roher und plumper Weise gearbeitet und ebenso ungeschlacht sind die Hände gebildet, die in jenem mit vielem Verständuiss und fein gegliedert erscheinen. Verschiedenheiten solcher Art bei sonst gleichen Formen können nur durch Nachbildung entstanden sein. Wie diese ausgefübrt wurde, ob durch ungeschickte Ausirbeitung eines schlecht gelungenen Abgusses, oder geradezu durch Nachschneiden des Siegelstempels, muss wol dahingestellt bleiben.

Ja es scheinen Omstände bestimmt darauf hinzudeuten, dass gerade dieses Siegel und auch diese Urkunde ron S. Georgen überbaupt als Vorbild gedient hat. Die Stiftungsurkunde zeigt als schnell in die Augen fallende Eigenthümlichkeit an allen vier Ecken rom Schreiber ausgesparte Räume: in dem rechts unten ist das Siegel Hartwigs angebracht, die indern sind leer, als wenn auch sie für ein Siegel bestimmt gewesen wären. Entweder hat der Schreiber noch nicht gewusst, wohin man das Siegel geben werde, oder er hat, was wol wahrscheinlicher ist, der Symmetrie halber nicht bloss an einer, sondern gleich au allen vier Ecken solche Räume gelassen. Und den analogen k'all dieser sonst ganz ungewöhnlichen Erscheinung constatirten wir bei unserer Urkunde über die S. Martinskirche; zwar ist hier uur ein Raum rechts oben frei gelassen, aber gewiss nicht aus hücksicht für ein Siegel, da für dasselbe ein mehr als genügender Platz unter dem Texte blieb, was man voraussehen musste. Ein zweiter Punct ist das Vorhandensein einer fast gleichlautenden Bannformel in beiden Urkunden. Konmt eine Bannformel überhaupt schon nicht häufig in dieser Urkundenregion vor, so dürfte eine beinahe wörtliche Uebereinstimmung in einer nicht gewöhn- 
lichen Fassung ${ }^{1}$ ) um so mehr als Zeicheu voll Verwandtschaft betrachtet werden.

Nun ist die Entstehungszeit dieses ganzen Machwerkes durch die Orkande über die Wiederherstellung und Neudotirung der S. Martinskirche von 1075 mit ziemlicher Sicherheit gegeben. Diese erwähnt ja die ursprüngliche Gründung und Ausstattung, die Verleihung der pfarrlichen Rechte und die Ablösung mit dem Gute Sulpa, und es ist gar kein Grund vorhanden daran zu zweifeln, dass wirklich unter Erzbischof Hartwig diese Kirche gegründet worden sei. Es wird darüber eine Aufzeichnung in Form eines Einzelactes ohne jede weitere Beglaubigung existirt haben. Om 1075 aber hatte man in der Werthschätzung der beglaubigten Urkunde bereits wieder einen Schritt nach vorwärts gethan, man, das heisst wol sicherlich in Gurk, dessen Bischof ja der zweite Gründer war, wollte eine förmliche Stiftungsurkunde. Das alte Stück wurde zur Vorlage für das Sachliche genommen, wol auch einiges der Fassung und vielleicht das Monatsdatum belassen ${ }^{2}$ ), für das andere jedoch und hauptsächlich zur möglichst gelungenen Nachahmung einer "feierlichen" Urkunde Hartwigs scheint als Muster die S. Georgener Stiftungsurkunde verwendet zu sein. Eine solche Benützung einer fremden, nicht dem eigenen Archive angehörigen Urkunde dürfte den Anschein der Unwahrscheinlichkeit, den sie etwa an sich trägt, verlieren, wenn man an deu bedeutsamen Antheil denkt, den der Bischof von Gurk an der Wiedererrichtung der S. Martinskirche nahm. So ward nun die Form dieser echten Urkunde Erzbischof Hartwigs hier nachgeahmt, der viereckige Raum getreulich offen gelassen, selbst die Schrift scheint in ihren misslungenen Schnörkeln an die diplomatische Minuskel der Vorlage sich anzulehnen; endlich ward das Siegel nach Möglichkeit nachgemacht und schliesslich auch die räthselhafte Jahreszahl als alterthümliches Relief hineingesetzt. Eine schlimme Absicht lag bei diesem Vorgehen fern, hat doch der Gurker Bischof selbst das alles, was darin gesagt ist, in seine Urkunde aufgenommen. Es ist eine neue Nüance dieser interessanten Fälle von Urkundenrenovationen.

Ihnen allen, die wir so kennen gelernt haben, gemeinsam ist das sichtliche Bestreben, durch das Siegel sich Glauben zu verschaffen

1) Sie erscheint noch einmal in der Beurkundung der weiteren Schenkungen der Gräfn Hemma an das von ibr gestiftete Nonnenkloster in Gurk durch Erzb. Balduin $104 \AA$ Jan. 6. Ankershofen, Gesch. Kärntens 2, Reg. u. Urk. 91 n. $4 \check{.}$.

$\Rightarrow$ Das Monatsdatum allein kommt auch in einer uns erbaltenen Tradition Hartwigs vor. Hauthaler-Richter, Die Salzburgischen Traditionscodices, Mitth. d. Instituts is, 88 n. 14, u. S. 874. 
und das Ansehen einer echten Urkunde zu gewinnen: im einen Fall drückte man ein echtes Siegel auf ein hundert Jahre jüngeres Elaborat, in einem zweiten bildete man das Siegel nach, in Göss hängte 1177 die Aebtissin ihr eigenes Siegel an ein auf Erabischof Gebhard lautendes Stück. Viel gleichgültiger ist man gegen die Schrift, nur beim letzten Fall scheint eine Nachahmung beabsichtigt, aber auch da, wie noch mehr bei den übrigen verräth uns die Schrift ganz unverhohlen die viel spätere Entstehung. Mehr Differenz zeigen die Formeln: hat man in Göss geradezu die alte Vorlage abgeschrieben, ja bei der Urkunde von S. Martin auf ein altes Formular mit Absicht zurückgegriffen, so entdecken uns im Gegentheil bei der Urkunde für die $\mathrm{S}$. Andreaskirche gerade die Formeln den Orsprung in Erzbischof Gebhards Zeit. Und doch treffen diese beiden letzten Stücke wieder im Streben zusammen, durch eine frei erfundene, recht weit zuräckliegende Datirung das angebliche, hohe Alter ihrer Entstehung zu documentiren; $j a$ in der letztgenannten hat man zudem noch durch die fingirte Unterzeichnung König Arnolfs ein weiteres Zeugniss dafür und um so stärkere Beglaubigung zu erstreben versucht. All das ist aber ein neuer Beweis für das bereits von Ficker betonte häufige Vorkommen solcher Fälle und wol auch ein Beweis dafür, mit welcher Naivetät man dabei vorgieng. Man war sich eben bewusst, in der Hauptsache in gutem Glauben und ohne Absicht der Fälschung zu handeln, aber man fand andererseits allerdings auch nichts besonderes darin, im einzelnen, wenn es leicht angieng, zur Bekräftigung und zur Erhöhung des Ansehens etwas dazu zu thun, wodurch man schon bewusst der Wahrbeit widersprach. Alle diese Dinge bewegen sich auf dem schlüpfrigen Zwischengebiet von voller Echtheit bis zu voller Fälschung. Nur in den günstigen Fällen, wo auch anderes Material zur Vergleichung und Prüfung vorhanden ist, wird es der Kritik gelingen können, die Entstehung und damit auch die Stufen der Glaubwürdigkeit solcher oft sehr sonderbarer Machwerke darzulegen. Aber immer werden wir uns hüten müssen, um einzelner Widersprüche willen gleich das Ganze in das Gebiet der Fälschung zu verweisen. 